\title{
THE NONEXISTENCE OF MAXIMUM SOLUTIONS OF VOLTERRA INTEGRAL EQUATIONS
}

\author{
H. E. GOLLWITZER AND R. A. HAGER
}

Abstract. It has been claimed for some time that the scalar Volterra integral equation of the second kind has a maximum solution under rather mild hypotheses. We present and discuss a counterexample to this claim.

Introduction. It has been claimed for some time that the scalar Volterra integral equation

$$
u(x)=f(x)+\int_{a}^{x} k(x, t, u(t)) d t, \quad x \in[a, b],
$$

always has a maximum solution when solutions are continuous and $f, k$ satisfy rather mild hypotheses [3], [4], [5]. Our purpose in this paper is to present and discuss a counterexample which may be of some independent interest. We begin with a few definitions and basic facts.

For simplicity we assume in (1) that: (i) $f$ is real-valued and continuous on the compact interval $I=[a, b]$; (ii) $k(x, t, u)$ is real-valued and continuous on $I \times I \times R$ where $R$ denotes the reals. Under these hypotheses Nohel [4] has shown that (1) has at least one continuous solution $u(x)$ on some interval $I_{\alpha}=[a, \alpha] \subseteq I$. By definition a solution $u_{M}(x)$ of (1) with the property that every solution $u(x)$ satisfies $u(x) \leqq u_{M}(x)$ on their common interval of existence is called the maximum solution of (1) on that subinterval. A minimum solution may be defined similarly. Clearly a maximum (minimum) solution is unique if it exists. With certain restrictions on $k(x, t, u)$, Sato [7] discussed the existence of a maximum solution of (1). It was announced by Nohel [4] (a proof was offered in [6]) that (1) always has a maximum (minimum) solution on some interval $I_{\alpha}$. In particular, if all solutions exist on $I$ then (1) has a maximum solution on $I$. That Nohel's claim is not true in general is a consequence of the following counterexample.

The counterexample. This counterexample may be of independent interest since explicit examples are not numerous in the theory of

Received by the editors February 9, 1970.

AMS 1969 subject classifications. Primary 4513, 4530.

Key words and phrases. Volterra integral equation, second kind, maximum solution, nonexistence, counterexample. 
integral equations. Consider the integral equation

$$
u(x)=-x \int_{-1}^{x} u(t)^{1 / 2} d t, \quad x \in[-1,1],
$$

where $u^{1 / 2}=|u|^{1 / 2} \operatorname{sgn} u$ for any real number $u$. Since the kernel $k(x, t, u)=-x u^{1 / 2}$ is continuous and satisfies $|k(x, t, u)| \leqq|u|^{1 / 2}$, it follows from elementary comparison considerations that every solution of (2) exists on $[-1,1]$. Also, the negative of a solution is again a solution. It is easily verified that the one parameter family $u(x, \delta)$, $0 \leqq \delta \leqq 1$, defined by

$$
u(x, \delta)=\frac{-x}{4}\left[\int_{-\delta}^{x} t^{1 / 2} d t\right]^{2}, \quad x \in[-\delta, \delta],
$$

and 0 elsewhere is a solution of $(2)$ on $[-1,1]$. The solutions $u_{1}(x)$ $\equiv u(x, 1), u_{2}(x) \equiv-u_{1}(x)$ are of particular interest and their graphs are indicated in the following figure.

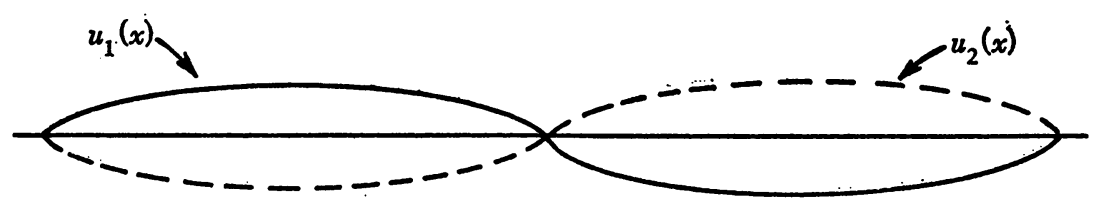

It will be shown later that $u_{2}(x) \leqq u(x) \leqq u_{1}(x)$ on $[-1,0]$ and $u_{1}(x) \leqq u(x) \leqq u_{2}(x)$ on $[0,1]$, where $u(x)$ is any solution of $(2)$.

If a maximum solution $u_{M}(x)$ exists, it must be given by

$$
\begin{aligned}
u_{M}(x) & =u_{1}(x) & & \text { for } x \in[-1,0], \\
& =u_{2}(x) & & \text { for } x \in[0,1] .
\end{aligned}
$$

Furthermore $u_{M}(x)$ must satisfy (2). However, for $0<x<1$ we would then have

$$
-x \int_{-1}^{x} u_{M}(t)^{1 / 2} d t=u_{M}(x)=u_{2}(x)=-x \int_{-1}^{x} u_{2}(t)^{1 / 2} d t
$$

or

$$
\int_{-1}^{0} u_{1}(t)^{1 / 2} d t=\int_{-1}^{0} u_{2}(t)^{1 / 2} d t
$$

This is a contradiction since $u_{2}=-u_{1}$ and $u_{2}$ is negative on $(-1,0)$. Thus (2) cannot have a maximum solution on $[-1,1]$.

It remains to establish the extremal properties of $u_{1}, u_{2}$ stated earlier. On $[-1,0)$ every solution of (2) is given by $u(x)=-x v(x)$ 
where $v(x)$ is a solution of the ordinary differential equation

$$
v^{\prime}=-x^{1 / 2} v^{1 / 2}, \quad v(-1)=0 .
$$

We will show that $v_{1}(x) \equiv-x^{-1} u_{1}(x)$ is the maximum solution of (3) on $[-1,0]$. The point $x=0$ is of no importance since it is a removable discontinuity for $v_{1}(x)$. From this it follows that $v_{2}(x)$ $\equiv-x^{-1} u_{2}(x)$ is the minimum solution of $(3)$ on $[-1,0]$. Since the solution curves of (3) are symmetric with respect to the origin, it is a simple matter to verify the extremal properties of $u_{1}, u_{2}$ stated earlier.

The proof that $v_{1}$ is the maximum solution of $[-1,0]$ is a simple modification of the usual arguments. We will sketch them here. Let $n$ be a positive integer and consider the initial value problem

$$
v^{\prime}=-x^{1 / 2}\left(v^{1 / 2}+1 / n\right), \quad v(-1)=0, \quad x \in[-1,0] .
$$

Since $-x^{1 / 2}>0$ on $[-1,0)$ it follows from standard arguments [2, p. 25] that $\lim _{n \rightarrow \infty} v(x, n)=v_{M}(x)$ uniformly on $[-1,0]$, where $v_{M}(x)$ is the maximum solution of $(3)$ and $v(x, n)$ is a solution of (4). However, (4) can be integrated implicitly to give

$$
2 v(x, n)^{1 / 2}-2 n^{-1} \ln \left(n v(x, n)^{1 / 2}+1\right)=-\int_{-1}^{x} t^{1 / 2} d t .
$$

Since $v(x, n) \leqq v(x, 1)$ it follows that $\lim _{n \rightarrow \infty} n^{-1} \ln \left(n v(x, n)^{1 / 2}+1\right)=0$. Taking limits in (5), we see that

$$
v_{M}(x)=\frac{1}{4}\left[\int_{-1}^{x} t^{1 / 2} d t\right]^{2}=v_{1}(x)
$$

and hence the counterexample is complete.

Conclusions. Roughly speaking, the proof offered by Nohel [6] fails since one generally cannot "patch together" solutions of integral equations and expect to obtain solutions on larger intervals. Indeed, this was demonstrated in the counterexample. This is in contrast to the situation in ordinary differential equations where "patching" techniques are applicable [1, pp. 45-47]. In view of this observation, it is clear that the important open problem is then to prescribe conditions on $f, k$ which imply that the maximum of any two solutions of (1) is again a solution. With such information available, one could use the results given by Walter $[8$, p. 24$]$ to formulate general theorems concerning the existence of a maximum solution of (1).

We note that for fixed $x$ the kernel $-x u^{1 / 2}$, of the counterexample, is nondecreasing in $u$ when $-1 \leqq x \leqq 0$ and nonincreasing in $u$ when 
$0 \leqq x \leqq 1$. It is well known that (1) has a maximum solution if $k(x, t, u)$ is nondecreasing in $u$ for each fixed $x, t$. However, the monotonicity of $k$ is not a necessary condition since the Volterra equation

$$
u(x)=x \int_{-1}^{x} u(t)^{1 / 2} d t, \quad x \in[-1,1],
$$

has a maximum solution on $[-1,1]$. This follows from the results of Sato [7]. It is an open question as to what extent, if any, Sato's kernel hypothesis can be weakened.

\section{REFERENCES}

1. E. A. Coddington and N. Levinson, Theory of ordinary differential equations, McGraw-Hill, New York, 1955. MR 16, 1022. \#1270.

2. P. Hartman, Ordinary differential equations, Wiley, New York, 1964. MR 30

3. V. Lakshmikantham and S. Leela, Differential and integral inequalities, Academic Press, New York, 1969.

4. J. A. Nohel, Some problems in nonlinear Volterra integral equations, Bull. Amer. Math. Soc. 68 (1962), 323-329. MR 26 \#2838.

5. - Problems in qualitative behavior of solutions of nonlinear Volterra equations, Proc. Advanced Sem. Nonlinear Integral Equations (Math. Res. Center, U. S. Army, Univ. of Wisconsin, Madison, Wis., 1963), Univ. of Wisconsin Press, Madison, Wis., 1964, pp. 191-214. MR 29 \#444.

6. - Qualitative behavior of solutions of nonlinear Volterra equations, Proc. NATO Advanced Study Inst. Stability Problems of Solutions of Differential Equations, (Padua, Italy, 1965), Oderisi, Gubbio, Italy, 1966, pp. 177-210.

7. T. Sato, Sur l'équation intégrale non linéaire de Volterra, Compositio Math. 11 (1953), 271-290. MR 15, 714.

8. W. Walter, Differential- und Integral-Ungleichungen, und ihre Anwendung bei Abschätzungs- und Eindeutigkeitsproblemen, Springer Tracts in Natural Philosophy, vol. 2, Springer-Verlag, Berlin and New York, 1964. MR 30 \#2302.

Drexel University, Philadelphia, Pennsylvania 19104

Cornell University, Ithaca, New York 14850 